\title{
An introduction to
}

\section{treatment

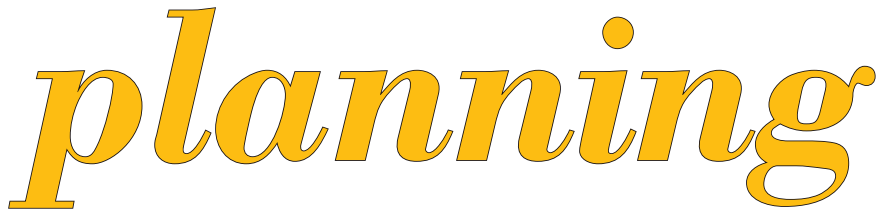

\section{How does renowned dentist Ashish B. Parmar' approach treatment planning for a new patient? He shares his approach for achieving long term success.}

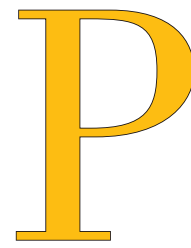

eople are living longer these

days. As such, they are keeping

their teeth for longer. This does

mean that treatment becomes

more complex - there can be

periodontal disease, wear of

teeth, moving or missing teeth. It is vital for the dentist to carry out a thorough and detailed initial dental assessment, spend adequate time analysing the diagnostic data, and formulate the ideal treatment plan for the patient based on their desires and budget. All the alternative treatment plans with the pros and cons should also be discussed so that the patient can make an informed decision.

This article will cover the systems I use and also teach, and which work very well for me in private practice.

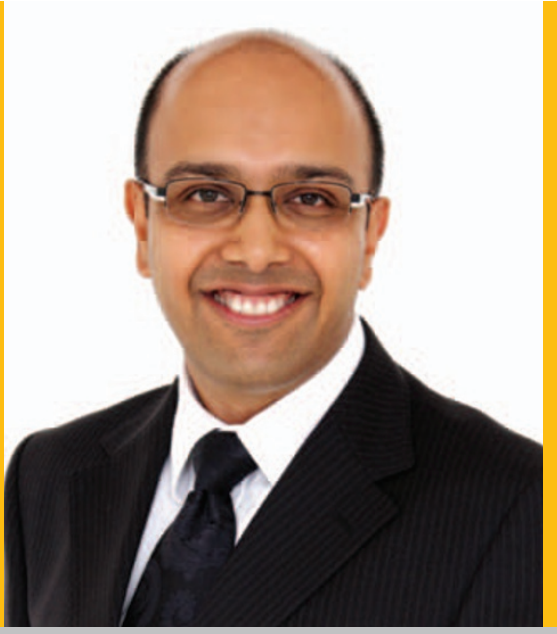

\section{Ash Parmar}

' Ash Parmar runs Smile Design By Ash in Chigwell, Essex with his wife Jyoti who is also a dentist. He is a renowned lecturer, widely published. Ash was one of the most featured dentists on the three series of Extreme Makeover UK programmes on Living TV. He has a special interest in cosmetic and restorative dentistry, implants and laser dentistry and his practice runs a variety of courses.

Email:ash@smiledesignbyash.co.uk www.theacademybyash.co.uk

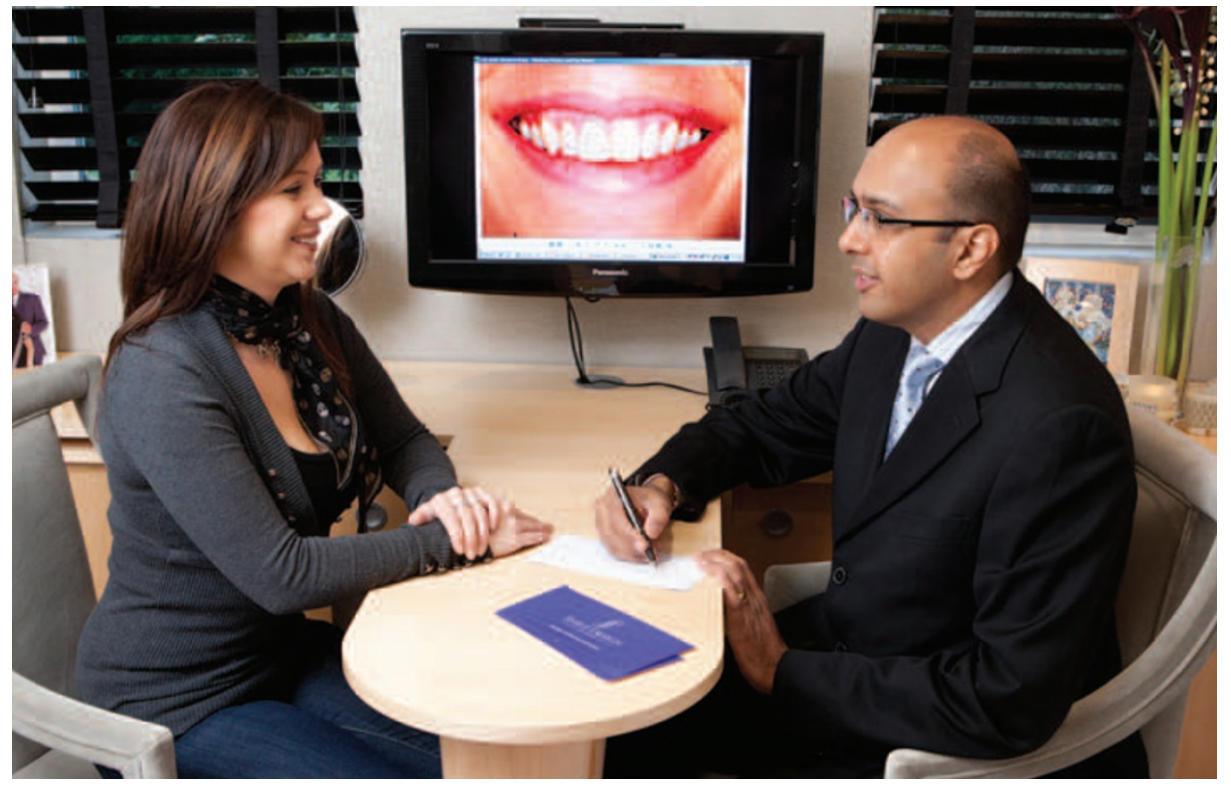

\section{Comprehensive dental assessment}

In our practice, we carry out a very detailed initial assessment, which takes the dentist approximately 1.5 hours to do. The following records are done:

• Visual assessment of posture and the head position

घ Clinical assessment of the TMJ and the facial muscles

- Detailed intra-oral examination (soft tissues, occlusion, teeth, periodontal, etc)

- Dental charting on the computer software (eg Software Of Excellence)

- 6 point pocket (periodontal) charting

$\square$ Smile analysis (detailed review of all the cosmetic parameters)

- Joint Vibration Analysis (TMJ evaluation)

- T Scan record (initial computerised bite record)

$\square$ Upper and lower alginates for baseline study models.

In addition, a full series of digital photographs, a dental panoramic tomograph, bitewing and appropriate periapical radiographs are also taken. A few other tips I would recommend at the assessment visit are:

$\square$ Co-discover the problems in the mouth. It is important to get the patient involved in the assessment so that they begin to understand the problems they have. I use the patient's clinical photographs on the screen that is mounted on the dental chair to educate and explain to the patient

- Understand the most important objectives the patient has, ie is the main motivating factor to have dental treatment health and function, or the appearance?

$\square$ It is also vital to overcome objections at this stage. For example if someone is scared of injections, then I would explain to them that we have different strengths of local anaesthetic (which is warmed up and injected very slowly). We would also show the patient the powerful topical anaesthetic that is placed on the gum for a few minutes before the injection is given. We explain about the relaxing music, calm environment, aromatherapy, etc. In this way, the patient feels more at ease about having the treatment in due course

- Give the patient relevant information sheets to take home before they leave this appointment. You can also reference the patient to certain specific pages on your website before they return to the case presentation appointment. This extra information will help the patient understand things better. 


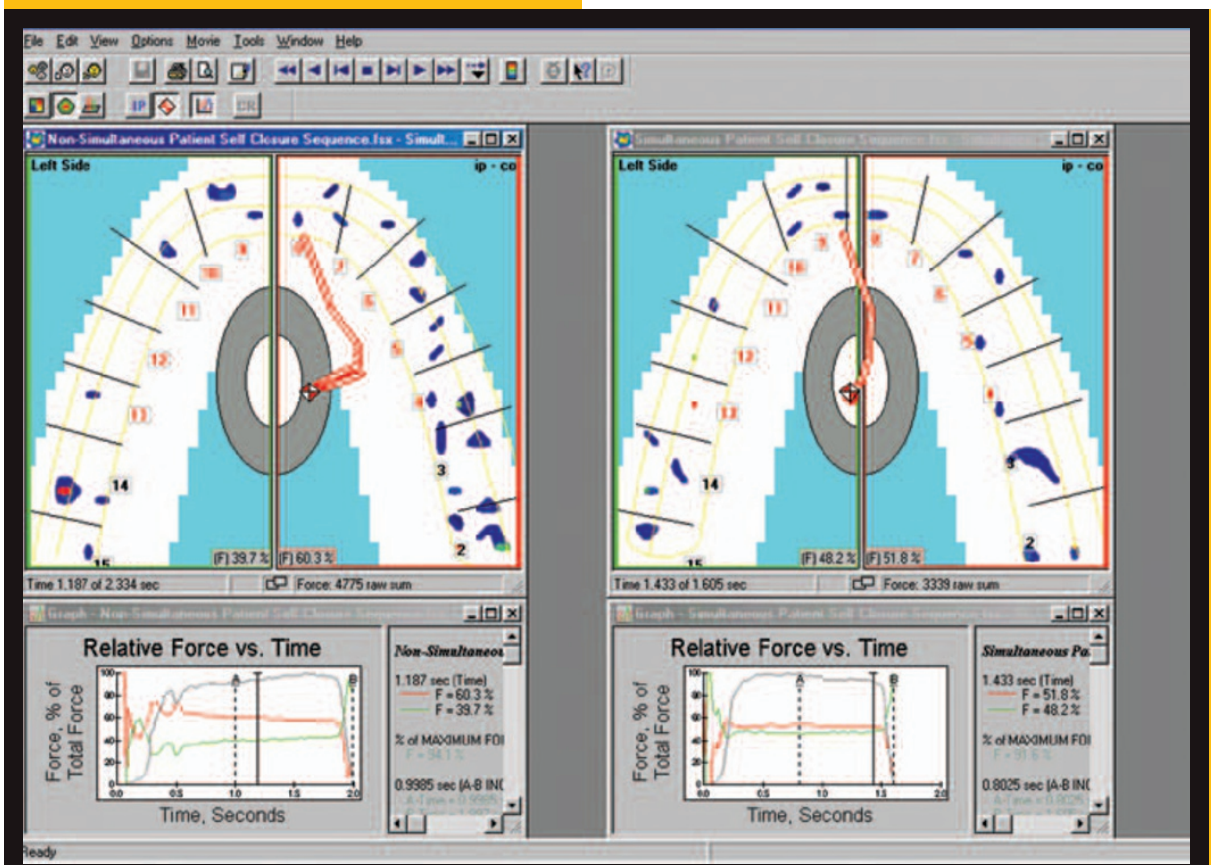

Fig. 1 T Scan occlusion record
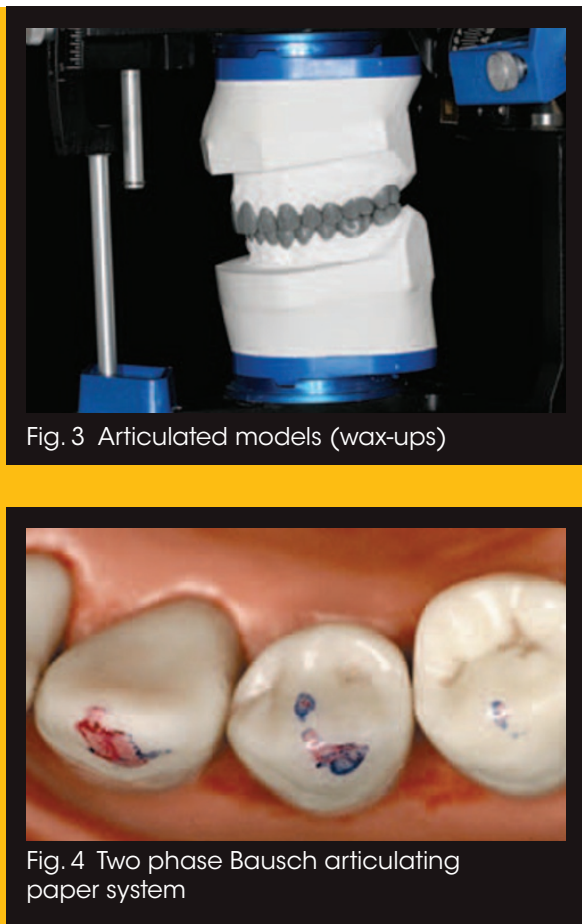

measurements that can be taken to help diagnose the pre-operative condition of the TMJ. In addition, I use computerised software called Joint Vibration Analysis, which is very good to make a quick and accurate differential diagnosis of the TMJ condition - this supports my clinical findings. If necessary, I can then refer the patient to a TMJ specialist for further advice and care before restorative treatment is carried out.

\section{Occlusion assessment}

I have a very detailed checklist of the occlusion assessment, which includes:

- Shimstock hold positions

- Contacts in centric occlusion and excursions

- Wear facets

- Abfraction

- Wear pattern analysis (attrition and erosion)

- Initial contact positions in centric relation, slides and occlusal interferences, etc.

I also highly recommend T Scan. This is a computerised system, which is probably the most accurate way in the world of assessing occlusion (Fig. 1). A special sensor helps capture detailed information on occlusal contacts within each cycle of teeth meeting together. This can then be reviewed in detail on the computer.

I find that looking at the study models (Fig.

2) (articulated if necessary) (Fig. 3) and the photographs of the patient also gives the dentist so many clues.

Regarding articulating paper choice (Fig. 4), I use the 'two phase articulation system' with Bausch articulating papers - using the 100 micron thicker blue paper first (this has transculase bonding agent and can mark even in 
the presence of some saliva), followed by red foil articulating paper which is only eight microns thick. This unique method is very accurate for marking occlusal contacts accurately.

\section{Treatment planning (after all the diagnostic data have \\ been collected)}

I use a very systematic approach in treatment planning. I am always mindful of the final result I am trying to achieve for the patient. I try and plan the treatment in the following way:

1. Ensuring the gums are very healthy (by seeing the hygienist, or periodontist if there is advanced gum disease)

2. Extraction of teeth with a poor prognosis

3. Assessing the bite and making adjustments if necessary to ensure there is harmony between the jaw joints, facial muscles and the teeth ie occlusal equilibration (I either use the MAP technique, or a leaf gauge, or T Scan during occlusal equilibration procedures)

4. Restoring broken or decayed teeth with cosmetic tooth coloured fillings (composite or porcelain restorations). Certain teeth may need root canal treatment and then a fibre post reinforced with composite as a core. The objective is to get the teeth decay free, and have secure and strong cores for any teeth that may require crowns

5. Improving the smile by using a whole range of cosmetic treatment options including teeth whitening, bonding, porcelain veneers and braces

\section{'I am always mindful of the}

\section{final result}

I am trying to achieve for the patient'

6. Replacing any missing teeth for improved function and aesthetics. Options may include bridges (conventional fixed or fibre reinforced composite bridges), implants and conventional or precision attachment dentures

7. Maintenance: recommendation of regular

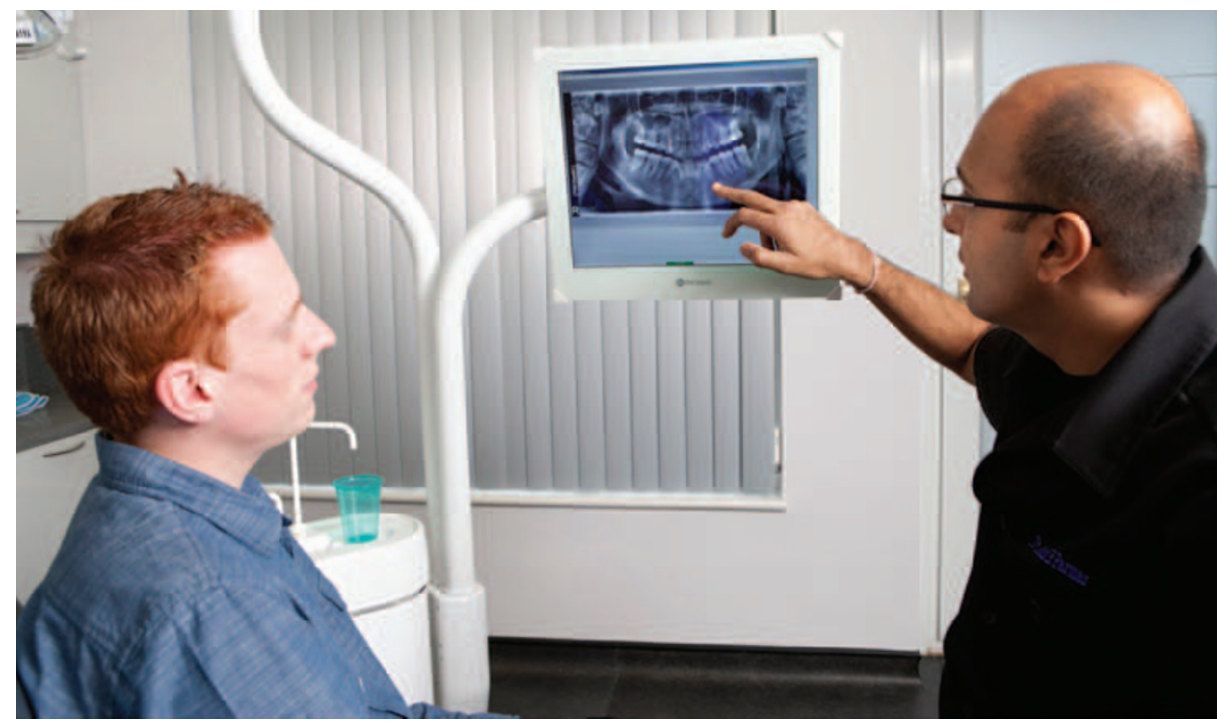

visits with the hygienist (based on the level of initial gum problems and the patient's oral hygiene) and an annual dental examination.

\section{The case presentation visit}

We invite the patient to return to the practice one week after the assessment appointment for a complimentary consultation visit to discuss the clinical findings.

In the meantime, the dentist and the treatment coordinator (TCO) need to plan for this important visit. After the ideal treatment plan has been made, and alternative treatment plans created, the dentist can review all this information with the TCO. The TCO then enters these treatment plans on to the computer.

The dentist needs to review the accuracy of the treatment plans with respect to the times required for the appointments, the fees, and the sequence of appointments.

The TCO then prints off the estimates and gets the consents ready, which the dentist should double-check. What I also do is initial the estimates so I know they are accurate and have been verified by me.

The other important task the TCO has to do is to create a PowerPoint presentation of the patients' photographs, OPG radiograph, annotations, etc. If you have treated similar cases and have a comparison case with photographs, this can also be added to the PowerPoint slides. This is a very professional, and more importantly, a visual way to communicate with the patient.

The dentist and TCO would also have planned carefully which demonstration aids (eg crown and bridge model, implants model, gum disease flip chart, GURU [computer software]) are required at the case presentation appointment. It is really important to be fully organised and clear what the dentist wants BEFORE the actual case presentation appointment.

\section{Dental reports \& consent documents}

I always provide a written, professional typed report to the patient. This gives the patient an excellent summary of all the clinical findings and explains the treatment plan and stages in which the treatment will be carried out. The patient is made clear of the diagnoses, the teeth that have a dubious long-term prognosis, and also a summary of the alternative treatment options.

We have a comprehensive range of detailed consent documents for all the following treatments:

- Wisdom teeth extractions

- Teeth whitening

- Smile makeovers

- Implants

- Sinus and bone grafting

$\square$ Tissue grafting

- Braces (eg 6 Month Smiles and The Inman Aligner).

\section{Summary}

The dentist has an ethical responsibility to offer the patient the ideal treatment plan based on their goals, needs/wants and budget. All alternative treatment options should be planned for and discussed, with written estimates. The TCO also has many important roles in supporting the dentist after the clinical assessment appointment and before the patient returns for the case presentation appointment.

Ash Parmar will be presenting the BDA Training essentials course Treatment Planning in London on 11 May 2012 alongside Laura Horton, one of the UK's leading treatment coordinator trainers. Contact the BDA Events Office on 0207563 4590 to book a place. For more information, visit www.bda.org/training 